\title{
Web of Science and Scopus are not global databases of knowledge
}

Jonathan P. Tennant

Email: jon.tennant.2@gmail.com

IGDORE, UK

https://orcid.org/0000-0001-7794-0218

\begin{abstract}
Both Web of Science and Scopus are critical components of our research ecosystem, providing the basis for university and global rankings, as well as for bibliometric research. However, both are structurally biased against research produced in non-western countries, non-English language research, and research from the arts, humanities and social sciences. This viewpoint emphasises the damage that these systematic inequities pose upon our global knowledge production systems, and the need to research funders to unite to form a more globally-representative, non-profit, community-controlled infrastructure for our global research knowledge pool.
\end{abstract}

There has never been a more pressing need for science to be working to address the major challenges that we face as a global society, as envisaged by the UN Sustainable Development Goals (1). Our climate is changing catastrophically and irreversibly, we are in the midst of yet another global pandemic (2), and we are facing resource distribution inequities like never before in the face of a booming global population. These are global challenges that affect us all, and therefore we need to ensure that the science we are using to help address them is truly globally representative $(3,4)$.

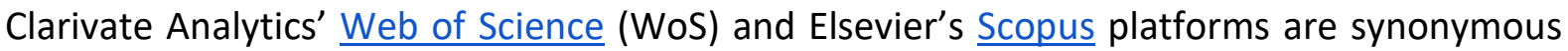
with data on international research. They are widely considered by the scholarly community as the two most trusted or authoritative sources of bibliometric data. They form the basis for virtually all of peer-reviewed knowledge on research across different disciplines. Below is a cartogram that illustrates a map of the world scaled by the number of publication authors per 
country, developed by Juan Pablo Alperin and Rodrigo Costas (Figure 1). The publication data are from Scopus, and reveal an alarmingly warped version of reality: research from Africa, South America, and major parts of Asia is almost non-existent.

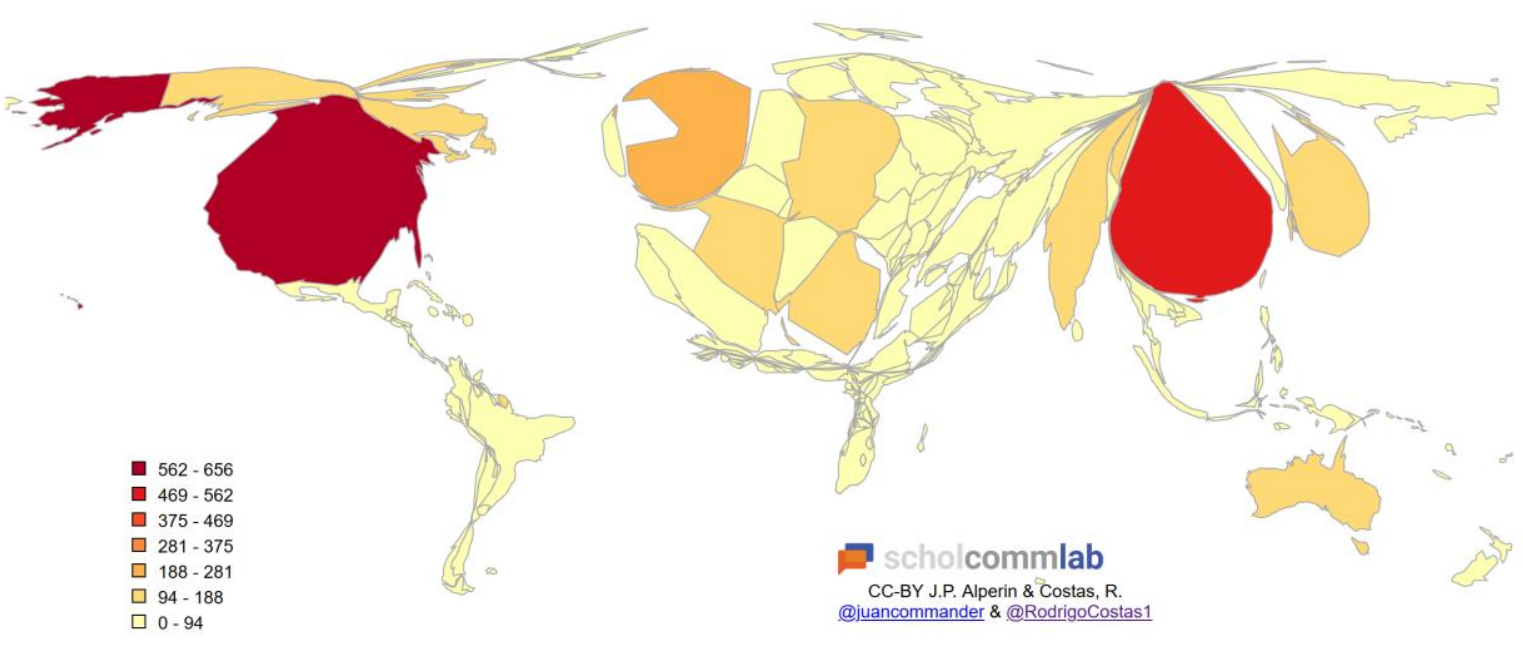

Figure 1. Map of the world scaled according to number of authors published in Scopus.

As the largest of their kind, WoS and Scopus are often hailed as "global" databases of knowledge and used widely for bibliographic research and academic assessments. This includes the creation of global higher education rankings or their adoption in tenure and promotion guidelines. WoS, for example, features strongly in the UK Research Excellence Framework 2021, and international league tables. Bibliographic data from Scopus represents more than $36 \%$ of assessment criteria in the popular Times Higher Education world university rankings.

Cameron Neylon and others recently criticised these rankings, demonstrating that the data sources underpinning them are heavily biased and incomplete $(5,6)$. This seems strange, given that we have imposed a critical dependence on them within our global scholarly ecosystem. Neither databases seem to do a fair, precise, or even reasonable job of being unbiased or globally representative. Instead, both platforms seem to discriminate against different forms of knowledge; and in particular, that which does not hail from the English-speaking western world. 
In general Scopus is larger and geographically broader than WoS. However, it still only covers a fraction of journal publishing outside of Europe and North America. This discrimination is especially visible in Asia. As of August 2017, Scopus reports a coverage of over 2,000 journals in the Asia-Pacific region, which it boasts as being " $230 \%$ more than the nearest competitor". Now, this might seem impressive, until you actually look at the data. In Indonesia alone, now the top country in the world for Open Access (OA) publishing (7), the national government's Garuda portal currently lists more than 9,000 journals published in the country. Still more than four times what Scopus indexes for the entire Asia-Pacific region. Similarly, in Japan, there are almost 3,000 journals currently listed on the national J-Stage platform.

Scopus currently lists 750 journals from Africa and the Middle East, 212\% more than the nearest competitor. African Journals Online alone indexes 524 journals. The Directory for Arabian Journals lists 319 journals. The Algerian Scientific Journal Platform indexes 510 journals. Iraqi Academic Scientific Journals lists 272 OA journals.

In Latin America, we see much the same story. Scopus claims to have about 700 journals listed from this region, $168 \%$ more than the nearest competitor. SciELO, which has been providing open infrastructure for Latin American health science journals since 1997, lists more than 1,700 active journals at the present. Redalyc also supports more than 550 social sciences journals in Latin America.

In terms of language preference, we see much the same pattern. As Ryan Regier stated in 2018: "For example, Scopus indexes more than 23,000 currently-published scholarly journals and only about 5,000 of these publish in languages other than English. (You can find this data in the Scopus Title List). Compare that with the Directory of Open Access Journals (DOAJ), who does a much better job with outreach in non-English countries, of their 11,000 journals, 6,000 publish in languages other than English. They have less than half the journals Scopus does and they still have more non-English Journals!"

A more recent study from 2019 confirmed the extent of this bias: "English dominates both WoS and Scopus $192.64 \%$ of the documents indexed in Scopus are in English and this percentage is even higher in the WoS with $95.37 \%$ compared to the second language with 
the highest number of documents in Scopus, Chinese, with $2.76 \%$ and the second language in WoS, Spanish, with 1.26\%)" (8).

Given that most nations on this planet do not speak English as a first language, unless imposed by colonialism, this clearly represents a hegemonic linguistic practice. This has consequences. First, non-native English-speaking authors may be required to spend parts of their research budgets, as well as a significant amount of time on effort, on making their work at a level that is at an 'acceptable' standard of English, a problem exacerbated by the exploitative publishor-perish system. While there are some clear advantages in homogenising the language of science, such a practice simultaneously erodes of the social and cultural contexts inherent in non-English languages.

Clarivate Analytics have taken some positive steps to broaden the scope of WoS, integrating the SciELO citation index and also through the creation of the Emerging Sources Index (ESI), which has allowed the inclusion of many more international titles into the service. Given the rich history of knowledge production in Latin America, the fact that it refers to this as the "emerging sources" index feels like quite the display of prejudice. It is only emergent in the sense that WoS did not index it previously, not because the research did not exist or was not well-established.

Together, these numbers highlight quite a mismatch between the industry leaders and the global reality, with a number of structural geographic discrepancies. In other parts of the world, this mismatch seems to be less of a problem. In western Europe, Scopus lists 11,000 journals, and 6,000 from North America. Other studies have also shown that these services tend to under-represent research from the arts, humanities, and social sciences (9). Combine this with the linguistic and geographic biases inherent in either database, it seems difficult to argue that this a quality issue.

One of the reasons for this skew might simply be that journals from the excluded are of varying or lower 'quality'. However, it is well known that legitimate publishing activities from the 'global south', such as Academic Journals, have an ethnocentric prejudice focused on them. Much of this perspective was created thanks to the infamous, and now largely academically discredited, 'predatory publishing' list (10). However, this remains a pernicious 
issue at the heart of our global scholarly communication system. First of all, defining the quality of both research and journals is beset with difficulties. A recent consensus definition on what constitutes a 'predatory publisher' did not seek to explicitly delimit legitimate and illegitimate publishing activities, but did highlight the complexity of this situation (11); even by these standards, the largest publishers like Elsevier could be classified as predatory.

Irrespective, the selection criteria for journals and the power imbalance this imposes upon the global research community should not be left to commercial third-parties such as WoS or Scopus. It should be the research community, including learned societies and institutes, who makes quality assessments and define the standards for journals and scholarly communication. WoS and Scopus are both commercial and for-profit services that, irrespective of their methods, have a fiduciary duty and accountability to that of their shareholders and investors. Not a duty to science, or to the public. The reality is that the global research community has out-sourced the critical functions of acting as custodians for our scholarly ecosystem to a handful of private companies. And not just that, but organisations with an incredible track record of harm to the scholarly community (12).

Both are shining beacons in the world of "platform capitalism" - with business models that not only predate but also outperform those of Google and Facebook in terms of profit (13). It is perhaps no wonder that these databases are so biased towards favouring research from western Europe and North America. WoS is owned by Clarivate Analytics, based in London, and Scopus by Elsevier, based primarily in Amsterdam and London. Their geographic location is not a coincidence here, two of the centres of historical western colonialism, of which the after-shocks are still being widely felt.

The widespread usage of both services continues to reinforce a western hegemony in global scientific endeavours. The result of this is a suppression on global innovation through reducing epistemic diversity in participation in the research process, and relegating specific forms of knowledge to the 'periphery' as a form of cognitive injustice. Exclusion of non-western knowledge from these databases dictates what we read, what we value, and we build upon. This in turn discriminates against the contexts of knowledge generation in those places, including invaluable cultural perspectives. For example, in Latin America, there is an incentive policy in Higher Education which aims to foster increasing internationalisation. This is 
understood as publishing in WoS and Scopus indexed journals. A consequence of this is that some researchers no longer focus on 'local' topics because they won't get published in 'international' journals. Virtually all journals publish important work at different levels, and are relevant to different elements of society. That this is supressed in systematic ways against specific forms of knowledge by WoS and Scopus should be a worrying pattern. I do not believe that either entity should be in control of defining what research is deemed significant or not, given that they intrinsically have little scientific interest in this matter.

This trend will continue as both Clarivate Analytics and Elsevier continue to extend their control over critical elements of scholarly infrastructure (14). The fact that Elsevier is also the leading publisher of scholarly content is not a coincidence, and amounts to one of the most significant conflicts of interest in the world (15). The very fabric of our knowledge society is in the ongoing process of been handed to for-profit enterprises. Knowledge infrastructures, academic cultures, and research practices have become subject to the maximisation of profits in the interests of a few and at the expense of everyone else.

The first simple step to resolve this problem is to simply stop all research using either platform, and for users and institutes to stop subscribing to them. By deconstructing or reducing the power of existing faulty elements of the scholarly ecosystem, funds and energy can be liberated and put to use in investing in a more open, non-profit, community-owned global scholarly communication infrastructure, which provides more efficient, effective, and representative information on the global knowledge landscape. This born-digital infrastructure should be truly equitable, comprehensive, and multi-lingual, facilitating fair participation in knowledge creation.

Decolonising scholarly communication is not something simple, and the primary focus must be on creating inclusive digital infrastructures that do not replicate the hegemony inherent in present systems. This requires synchrony in both reducing the power status of those existing systems, while simultaneously amplifying other voices that have previously been marginalised to occupy such a space. International research funding bodies here have a key responsibility to unite to help achieve this in the context of the UN Sustainable Development Goals. This promises to address the geopolitical impact that existing systematic discrimination has on 
knowledge production (16), and further the inclusion and representation of marginalized research demographics within the global research landscape.

\section{Acknowledgements}

Thank you to Andy Nobes, Bárbara Rivera-López, and Asura Enkhbayar for valuable discussions and input to an earlier version of this article.

\section{References}

1. Griggs D, Stafford-Smith M, Gaffney O, Rockström J, Öhman MC, Shyamsundar P, et al. Sustainable development goals for people and planet. Nature. 2013 Mar;495(7441):305-7.

2. Dunleavy DJ. Coronavirus As Impetus For a Lasting Change in Research Culture [Internet]. SocArXiv; 2020 Mar [cited 2020 Mar 5]. Available from: https://osf.io/2ryt3

3. Archambault É, Campbell $D$, Gingras $Y$, Larivière V. Comparing bibliometric statistics obtained from the Web of Science and Scopus. Journal of the American Society for Information Science and Technology. 2009;60(7):1320-6.

4. Ciarli T, Rafols I, Llopis O. The under-representation of developing countries in the main bibliometric databases: a comparison of rice studies in the Web of Science, Scopus and CAB Abstracts. Proceedings of the science and technology indicators conference 2014 Leiden: "Context Counts: Pathways to Master Big and Little Data." 2014;97-106.

5. Huang C-K (Karl), Neylon C, Brookes-Kenworthy C, Hosking R, Montgomery L, Wilson K, et al. Comparison of bibliographic data sources: Implications for the robustness of university rankings. bioRxiv. 2019 Sep 1;750075.

6. Selten F, Neylon C, Huang C-K, Groth P. A Longitudinal Analysis of University Rankings. arXiv:190810632 [physics] [Internet]. 2020 Jan 20 [cited 2020 Mar 12]; Available from: http://arxiv.org/abs/1908.10632

7. Noorden RV. Indonesia tops open-access publishing charts. Nature [Internet]. 2019 May 15 [cited 2020 Mar 12]; Available from: https://www.nature.com/articles/d41586019-01536-5

8. Vera-Baceta M-A, Thelwall M, Kousha K. Web of Science and Scopus language coverage. Scientometrics. 2019 Dec 1;121(3):1803-13. 
9. Martín-Martín A, Orduna-Malea E, Delgado López-Cózar E. Coverage of highly-cited documents in Google Scholar, Web of Science, and Scopus: a multidisciplinary comparison. Scientometrics. 2018 Sep 1;116(3):2175-88.

10. Eve MP, Priego E. Who is Actually Harmed by Predatory Publishers? 1. 2017 Aug $13 ; 15(2): 755-70$.

11. Grudniewicz A, Moher D, Cobey KD, Bryson GL, Cukier S, Allen K, et al. Predatory journals: no definition, no defence. Nature. 2019 Dec;576(7786):210-2.

12. Tennant JP. Democratising knowledge: A report on the scholarly publisher Elsevier [Internet]. Education International; 2018. Available from: https://bit.ly/2PPjwRK

13. Mirowski P. The future(s) of open science. Soc Stud Sci. 2018 Apr 1;48(2):171-203.

14. Posada A, Chen G. Inequality in Knowledge Production: The Integration of Academic Infrastructure by Big Publishers. In: Cha L, Mounie P, editors. ELPUB 2018 [Internet]. Toronto, Canada; 2018 [cited 2019 Jan 24]. Available from: https://hal.archivesouvertes.fr/hal-01816707

15. Tennant J, Brembs B. RELX referral to EU competition authority [Internet]. Zenodo; 2018 Oct [cited 2018 Dec 12]. Available from: https://zenodo.org/record/1472045\#.XBEeoWjolPY

16. Paasi A. Academic Capitalism and the Geopolitics of Knowledge. In: The Wiley Blackwell Companion to Political Geography [Internet]. John Wiley \& Sons, Ltd; 2015 [cited 2019 Mar 1]. p. 507-23. Available from:

https://onlinelibrary.wiley.com/doi/abs/10.1002/9781118725771.ch37 